J. Korean Math. Soc. 48 (2011), No. 6, pp. 1189-1201

http://dx.doi.org/10.4134/JKMS.2011.48.6.1189

\title{
ALMOST PRINCIPALLY SMALL INJECTIVE RINGS
}

\author{
YUEMING XIANG
}

\begin{abstract}
Let $R$ be a ring and $M$ a right $R$-module, $S=\operatorname{End}_{R}(M)$. The module $M$ is called almost principally small injective (or $A P S$ injective for short) if, for any $a \in J(R)$, there exists an $S$-submodule $X_{a}$ of $M$ such that $l_{M} r_{R}(a)=M a \oplus X_{a}$ as left $S$-modules. If $R_{R}$ is an $A P S$-injective module, then we call $R$ a right $A P S$-injective ring. We develop, in this paper, $A P S$-injective rings as a generalization of $P S$ injective rings and $A P$-injective rings. Many examples of $A P S$-injective rings are listed. We also extend some results on $P S$-injective rings and $A P$-injective rings to $A P S$-injective rings.
\end{abstract}

\section{Introduction}

Let $R$ be a ring. A right ideal $I$ of $R$ is called small if, for every proper right ideal $K$ of $R, K+I \neq R$. Recall that a ring $R$ is right principally small injective (or $P S$-injective) (resp. $P$-injective, small injective, mininjective) if every $R$ homomorphism $f: I \rightarrow R$, for every principally small (resp. principally, small, minimal) right ideal $I$, can be extended to $R$. The detailed discussion of $P$ injective, small injective and mininjective rings can be found in $[2,3,4,8,9$, $10,12]$. The concept of $P S$-injective rings was first introduced in [14] as a generalization of $P$-injective rings and small injective rings. It was shown that every right $P S$-injective ring is also right mininjective. In [11], Page and Zhou introduced $A P$-injectivity and $A G P$-injectivity of modules and rings. Given a right $R$-module $M, S=\operatorname{End}_{R}(M)$. The module $M$ is called $A P$-injective if, for any $a \in R$, there exists an $S$-submodule $X_{a}$ of $M$ such that $l_{M} r_{R}(a)=M a \oplus X_{a}$ as left $S$-modules. The module $M$ is called $A G P$-injective if, for any $0 \neq a \in R$, there exists a positive integer $n=n(a)$ and an $S$-submodule $X_{a}$ of $M$ such that $a^{n} \neq 0$ and $l_{M} r_{R}\left(a^{n}\right)=M a^{n} \oplus X_{a}$ as left $S$-modules. A ring $R$ is called right $A P$-injective (resp. $A G P$-injective) if $R_{R}$ is an $A P$-injective (resp. AGPinjective) module. Many of the results on right $P$-injective rings were obtained for the two classes of right $A P$-injective rings and right $A G P$-injective rings. In [17], Zhou continued the study of left $A P$-injective rings and left $A G P$-injective rings with various chain conditions.

Received June 19, 2010.

2010 Mathematics Subject Classification. 16N20, 16P40, 16D50.

Key words and phrases. APS-injective modules (rings), trivial extensions. 
In the present paper, we say that a right $R$-module $M$ is $A P S$-injective if, for any $a \in J(R)$, there exists an $S$-submodule $X_{a}$ of $M$ such that $l_{M} r_{R}(a)=$ $M a \oplus X_{a}$ as left $S$-modules. A ring $R$ is called right $A P S$-injective if $R_{R}$ is an $A P S$-injective module. Similarly, we can define a left $A P S$-injective ring. Some examples are listed to show that $A P S$-injective rings are the proper generalization of $P S$-injective rings and $A P$-injective rings. It is also shown that there are many similarities between $A P$-injective rings and $A P S$-injective rings. In light of this fact, some results on $P S$-injective rings and $A P$-injective rings are as the corollaries of our results, respectively.

Throughout $R$ is an associative ring with identity and all modules are unitary. $J=J(R), \operatorname{soc}\left(R_{R}\right)$ and $Z\left(R_{R}\right)$ denote the Jacobson radical, right socle and right singular ideal of $R$, respectively. For a right $R$-module $M$, let $S=\operatorname{End}_{R}(M)$, then we have an $(S, R)$-bimodule $M$. If $X$ is a subset of $R$, the right (left) annihilator of $X$ in $R$ is denoted by $r_{R}(X)\left(l_{R}(X)\right)$. We write $a \in L-I$ to indicate that $a \in L$ but $a \notin I$ and $N \leq^{e} M$ to indicate that $N$ is an essential submodule of $M$. The notation $M^{n}$ stands for the direct sum of $n$ copies of the module $M$, written as column matrices. For the usual notations we refer the reader to [1], [6] and [10].

\section{Examples and basic properties}

Definition 2.1. Let $M$ be a right $R$-module, $S=\operatorname{End}_{R}(M)$. The module $M$ is called almost principally small injective (or APS-injective for short) if, for any $a \in J(R)$, there exists an $S$-submodule $X_{a}$ of $M$ such that $l_{M} r_{R}(a)=M a \oplus X_{a}$ as left $S$-modules. If $R_{R}$ is an $A P S$-injective module, then we call $R$ a right $A P S$-injective ring. Similarly, we can define the concept of left $A P S$-injective rings.

For an $R$-module $N$ and a submodule $P$ of $N$, we will identify $\operatorname{Hom}_{R}(N, M)$ with the set of homomorphisms in $\operatorname{Hom}_{R}(P, M)$ that can be extended to $N$, and hence $\operatorname{Hom}_{R}(N, M)$ can be seen as a left $S$-submodule of $\operatorname{Hom}_{R}(P, M)$.

Lemma 2.2. Let $M_{R}$ be a module, $S=\operatorname{End}_{R}(M)$ and $a \in J(R)$.

(1) If $l_{M} r_{R}(a)=M a \oplus X$ for some $X \subseteq M$ as left $S$-modules, then $\operatorname{Hom}_{R}(a R, M)=\operatorname{Hom}_{R}(R, M) \oplus \Gamma$ as left $S$-modules, where $\Gamma=\{f \in$ $\left.\operatorname{Hom}_{R}(a R, M): f(a) \in X\right\}$.

(2) If $\operatorname{Hom}_{R}(a R, M)=\operatorname{Hom}_{R}(R, M) \oplus Y$ as left $S$-modules, then $l_{M} r_{R}(a)$ $=M a \oplus X$ as left $S$-modules, where $X=\{f(a): f \in Y\}$.

(3) $M a$ is a direct summand of $l_{M} r_{R}(a)$ as left $S$-modules if and only if $\operatorname{Hom}_{R}(R, M)$ is a direct summand of $\operatorname{Hom}_{R}(a R, M)$ as left $S$-modules.

Proof. The proof is similar to that of [11, Lemma 1.2].

From Lemma 2.2, we have the following corollary.

Corollary 2.3. Let $M_{R}$ be a module and $a \in J(R)$. Then $l_{M} r_{R}(a)=M a$ if and only if every $R$-homomorphism of a $R$ into $M$ extends to $R$. 
Remark 2.4. (1) Obviously, right $P S$-injective modules are right $A P S$-injective. But the converse is false in general. For example, let $R=\left(\begin{array}{cc}F & F \\ 0 & F\end{array}\right)$ with $F$ a field and $M_{R}=\left(\begin{array}{ll}0 & F \\ 0 & F\end{array}\right)$. Then $M$ is right $A P S$-injective but not right $P S$-injective. In fact, choose $0 \neq x \in F$. Then $a=\left(\begin{array}{cc}0 & x \\ 0 & 0\end{array}\right) \in J(R)$ and $l_{M} r_{R}(a)=M \neq$ $M a=0$. By the preceding corollary, $M$ is not right $P S$-injective. Note that $J(R)=\left(\begin{array}{ll}0 & F \\ 0 & 0\end{array}\right)$. Thus, $l_{M} r_{R}(a)=M a \oplus M$ for any $a \in J(R)$. Therefore, $M$ is right $A P S$-injective.

(2) Right $A P$-injective modules are right $A P S$-injective.

(3) Right $A P S$-injective rings are right almost mininjective [13] (A ring $R$ is called right almost mininjective if, for any minimal right ideal $k R$ of $R$, there exists an $S$-submodule $X_{k}$ of $R$ such that $l_{R} r_{R}(k)=R k \oplus X_{k}$ as left $S$-modules). In fact, in view of [6, Lemma 10.22], every minimal right ideal of $R$ is either nilpotent or a direct summand of $R$.

Example 2.5. The three examples of [11, Examples 1.5] are commutative $A P S$-injective but not $P S$-injective.

Example 2.6. Let $R=\mathbb{Z}$ be the ring of integers. Then $R$ is $A P S$-injective but not $A G P$-injective.

Example 2.7. Let $K$ be a field and $L$ be a proper subfield of $K$ such that $\rho: K \rightarrow L$ is an isomorphism, e.g., let $K=F\left(y_{1}, y_{2}, \ldots\right)$ with $F$ a field, $\rho\left(y_{i}\right)=y_{i+1}$ and $\rho(c)=c$ for all $c \in F$. Let $K\left[x_{1}, x_{2} ; \rho\right]$ be the ring of twisted right polynomials over $K$ where $k x_{i}=x_{i} \rho(k)$ for all $k \in K$ and for $i=1,2$. Set $R=K\left[x_{1}, x_{2} ; \rho\right] /\left(x_{1}^{2}, x_{2}^{2}\right)$. In view of [3, Example 1 and Proposition 1], $R$ is a left $A G P$-injective but not $A P S$-injective.

Theorem 2.8. Let $R$ be a right $A P S$-injective ring. Then.

(1) $J(R) \subseteq Z\left(R_{R}\right)$.

(2) $\operatorname{soc}\left(R_{R}\right) \subseteq r_{R}(J)$.

Proof. (1) Take any $a \in J(R)$. If $a \notin Z\left(R_{R}\right)$, then there exists a nonzero right ideal $I$ of $R$ such that $r_{R}(a) \cap I=0$. So there exists $b \in I$ such that $a b \neq 0$. Note that $a b \in J(R)$, by hypothesis, there exists $0 \neq u \in a b R$ such that $l_{R} r_{R}(u)=R u \oplus X_{u}$, where $X_{u} \subseteq{ }_{R} R$. Write $u=a b c$ for some $c \in R$. If $t \in r_{R}(a b c)$, then $a b c t=0$, implying $c t \in r_{R}(a b)=r_{R}(b)$ since $r_{R}(a) \cap I=0$. Hence, $(b c) t=b(c t)=0$, and so $t \in r_{R}(b c)$. This shows that $r_{R}(b c)=r_{R}(a b c)$. Note that $b c \in l_{R} r_{R}(b c)=l_{R} r_{R}(a b c)=R u \oplus X_{u}$. Write $b c=d a b c+x$, where $d a b c \in R u-X_{u}$ and $x \in X_{u}-R u$. Then $x=(1-d a) b c$, and so $b c=(1-d a)^{-1} x \in X_{u}$ since $1-d a$ is invertible, contradicting with $d a b c \in R u-X_{u}$.

(2) Let $k R$ be a simple right ideal of $R$. Suppose $j k \neq 0$ for some $j \in J(R)$, then $r_{R}(j k)=r_{R}(k)$. Note that $j k \in J(R)$ and $R$ is right $A P S$-injective. Then there exists a left ideal $X_{j k}$ of $R$ such that $l_{R} r_{R}(j k)=R j k \oplus X_{j k}$. Since $k \in l_{R} r_{R}(j k)$, write $k=r j k+x$, where $r j k \in R j k-X_{j k}$ and $x \in X_{j k}-R j k$. Then $x=(1-r j) k$, and hence $k=(1-j k)^{-1} x \in X_{j k}$ since $1-j k$ is invertible, contradicting with $r j k \in R j k-X_{j k}$. 
The following example shows that a right mininjective ring need not be right APS-injective.

Example 2.9. Let $R$ be the ring of all $\mathbb{N}$-square upper triangular matrices over a field $F$ that are constant on the diagonal and have only finitely many nonzero entries off the diagonal. By [16, Example 1.7], $\operatorname{soc}\left(R_{R}\right)=Z\left(R_{R}\right)=0$ and $J(R) \neq 0$. So $R$ is right mininjective. However, $R$ is not right $A P S$ injective by Theorem 2.8(1).

A ring $R$ is called semiregular if $R / J(R)$ is von Neumann regular and idempotents lift modulo $J(R)$, equivalently if, for any $a \in R$, there exists $e^{2}=e \in R a$ such that $a(1-e) \in J(R)$ (cf. [10, Lemma B.40]).

Proposition 2.10. If $R$ is semiregular, then $R$ is right AP-injective if and only if $R$ is right $A P S$-injective.

Proof. It is enough to prove sufficient condition. Since $R$ is semiregular, for any $a \in R, R a=R e \oplus R b$, where $e^{2}=e \in R$ and $b \in J(R)$. By hypothesis, $l_{R} r_{R}(b)=R b \oplus X_{b}$ for some left ideal $X_{b}$ of $R$. Then $R a \oplus X_{b}=R e \oplus R b \oplus$ $X_{b}=l_{R}(1-e) \oplus l_{R} r_{R}(b)=l_{R}\left((1-e) R \oplus r_{R}(b)\right)=l_{R}\left(r_{R}(R e) \oplus r_{R}(R b)\right)=$ $l_{R} r_{R}(R e \oplus R b)=l_{R} r_{R}(R a)=l_{R} r_{R}(a)$. Therefore, $R$ is right $A P$-injective.

Remark 2.11. There exists a ring that is semiregular but not right APSinjective. Let $R=\left(\begin{array}{cc}\mathbb{Z}_{2} & \mathbb{Z}_{2} \\ 0 & \mathbb{Z}_{2}\end{array}\right)$, where $\mathbb{Z}_{2}$ is the ring of integers modulo 2. Then $J(R)=\left(\begin{array}{cc}0 & \mathbb{Z}_{2} \\ 0 & 0\end{array}\right)$, and $Z\left(R_{R}\right)=0$. By Theorem 2.8, $R$ is not right $A P S$-injective. But $R / J(R) \cong\left(\begin{array}{cc}\mathbb{Z}_{2} & 0 \\ 0 & \mathbb{Z}_{2}\end{array}\right)$ is von Neumann regular and any idempotent of $R / J(R)$ can be lifted to $R$, so $R$ is semiregular.

By Proposition 2.10 and [11, Theorem 2.16], we have the following result.

Corollary 2.12. If $R$ is a semiperfect and right $A P S$-injective ring, then $R=R_{1} \times R_{2}$, where $R_{1}$ is semisimple and every simple right ideal of $R_{2}$ is nilpotent.

Clearly, a semiprimitive ring $(J(R)=0)$ is left and right $A P S$-injective. But the converse is not true as Example 2.5. Next, we shall consider when a right $A P S$-injective ring is semiprimitive. Following [7], $\mathrm{A}$ ring $R$ is called a right $J-P P$ ring if $a R$ is projective for any $a \in J(R)$.

Proposition 2.13. Let $R$ be a ring. Then the following are equivalent:

(1) $R$ is semiprimitive.

(2) $R$ is right $J-P P$ and right $A P S$-injective.

(3) $R$ is a right APS-injective ring whose every simple singular right $R$ module is $P S$-injective.

Proof. $(1) \Rightarrow(2)$ and $(1) \Rightarrow(3)$ are trivial.

$(2) \Rightarrow(1)$. Suppose $0 \neq a \in J(R)$. Since $R$ is right $J-P P, a R$ is projective. So the exact sequence $0 \rightarrow r_{R}(a) \rightarrow R \rightarrow a R \rightarrow 0$ splits. Then $r_{R}(a)=e R$ for some $e^{2}=e \in R$. It follows that $l_{R} r_{R}(a)=l_{R}(e R)=R(1-e)$. Note that 
$R$ is also right $A P S$-injective, so there exists a left ideal $X_{a}$ of $R$ such that $l_{R} r_{R}(a)=R a \oplus X_{a}$. Then $R a$ is a direct summand of $R(1-e)$, and hence a direct summand of ${ }_{R} R$, which implies $a=0$, a contradiction.

$(3) \Rightarrow(1)$. We first show that $J \bigcap Z\left(R_{R}\right)=0$. Take any $b \in J \bigcap Z\left(R_{R}\right)$. If $b \neq 0$, then $r_{R}(b)+R b R$ is an essential right ideal of $R$. If $r_{R}(b)+R b R \neq R$, there exists a maximal essential right ideal $T$ of $R$ such that $r_{R}(b)+R b R \subseteq$ $T$. By hypothesis, $R / T$ is $P S$-injective. Note that $r_{R}(b) \subseteq T$, then the $R$ homomorphism $f: b R \rightarrow R / T$ by $b r \mapsto r+T$ is well defined. So $f=(c+T)$. for some $c \in R$. Then $f(b)=1+T=c b+T$. Note that $c b \in R b R \subseteq T$, so $1 \in T$, a contradiction. This proves that $r_{R}(b)+R b R=R$, and hence $r_{R}(b)=R$ because $R b R$ is a small ideal of $R$. This implies $b=0$, which is required contradiction. Therefore, $J(R)=J \cap Z\left(R_{R}\right)=0$ by Theorem $2.8(1)$.

Now we construct a right $A P S$-injective ring that is not left $A P S$-injective.

Example 2.14. Let $R=\left(\begin{array}{cc}K & K \\ 0 & A\end{array}\right)$, where $K=\mathbb{Z}_{2}$ and

$$
A=\left\{\left(a_{1}, a_{2}, \ldots, a_{n}, a, a, \ldots\right) \mid a, a_{1}, a_{2}, \ldots \in K, n \in \mathbb{N}\right\} .
$$

If $k \in K$ and $\left(a_{1}, a_{2}, \ldots, a_{n}, a, a, \ldots\right) \in A$, let $k \cdot\left(a_{1}, a_{2}, \ldots, a_{n}, a, a, \ldots\right)=k a$.

Following [2, Example 1], $R$ is right $P$-injective, and hence right $A P S$ injective. But $J(R)=\left(\begin{array}{ll}0 & K \\ 0 & 0\end{array}\right) \neq 0$, so $R$ is not semiprimitive. We claim that $R$ is not left $A P S$-injective. By Proposition 2.13, it is enough to show that every simple singular left $R$-module is $P S$-injective. In fact, $M=\left(\begin{array}{cc}K & K \\ 0 & \mathbb{Z}_{2}^{(\mathbb{N})}\end{array}\right)$ is the unique maximal essential right ideal of $R$, where

$$
\mathbb{Z}_{2}^{(\mathbb{N})}=\left\{\left(a_{1}, a_{2}, \ldots, a_{n}, 0,0, \ldots\right) \mid a_{1}, a_{2}, \ldots \in K, n \in \mathbb{N}\right\} .
$$

In view of $[15$, p. 5$], \bar{R}=R / M$ is left $P$-injective, and hence left $P S$-injective.

Proposition 2.15. If $R$ is a right $A P S$-injective ring and $R / \operatorname{soc}\left(R_{R}\right)$ satisfies the ACC on right annihilators, then $J(R)$ is nilpotent.

Proof. Write $S=\operatorname{soc}\left(R_{R}\right)$ and $\bar{R}=R / S$. For any sequence $a_{1}, a_{2}, a_{3}, \ldots \in$ $J(R)$, there is an ascending chain

$$
r_{\bar{R}}\left(\overline{a_{1}}\right) \subseteq r_{\bar{R}}\left(\overline{a_{2}} \overline{a_{1}}\right) \subseteq r_{\bar{R}}\left(\overline{a_{3}} \overline{a_{2}} \overline{a_{1}}\right) \subseteq \cdots,
$$

by hypothesis, there exists a positive integer $m$ such that

$$
r_{\bar{R}}\left(\overline{a_{m}} \cdots \overline{a_{2}} \overline{a_{1}}\right)=r_{\bar{R}}\left(\overline{a_{m+k}} \cdots \overline{a_{m}} \cdots \overline{a_{2}} \overline{a_{1}}\right), k=1,2, \ldots
$$

Since $a_{n+1} a_{n} \cdots a_{1} \in J(R) \subseteq Z\left(R_{R}\right)$ by Theorem 2.8(1), $r_{R}\left(a_{n+1} a_{n} \cdots a_{1}\right)$ is the essential right ideal of $R$. Then $S \subseteq r_{R}\left(a_{n+1} a_{n} \cdots a_{1}\right)$.

Now we prove that

$$
r_{\bar{R}}\left(\overline{a_{n}} \cdots \overline{a_{2}} \overline{a_{1}}\right) \subseteq r_{R}\left(a_{n+1} a_{n} \cdots a_{1}\right) / S \subseteq r_{\bar{R}}\left(\overline{a_{n+1}} \overline{a_{n}} \cdots \overline{a_{1}}\right) .
$$

In fact, for any $b+S \in r_{\bar{R}}\left(\overline{a_{n}} \cdots \overline{a_{2}} \overline{a_{1}}\right), a_{n} \cdots a_{1} b \in S$. Then $a_{n+1} a_{n} \cdots a_{1} b=$ 0 because $S \subseteq r_{R}\left(a_{n+1}\right)$. So $b \in r_{R}\left(a_{n+1} a_{n} \cdots a_{1}\right)$, and hence $b+S \in$ $r_{R}\left(a_{n+1} a_{n} \cdots a_{1}\right) / S$. But the second inclusion is clear. 
Since $r_{\bar{R}}\left(\overline{a_{m}} \cdots \overline{a_{2}} \overline{a_{1}}\right)=r_{\bar{R}}\left(\overline{a_{m+2}} \overline{a_{m+1}} \cdots \overline{a_{2}} \overline{a_{1}}\right)$, by (1),

$$
r_{R}\left(a_{m+1} a_{m} \cdots a_{1}\right) / S=r_{R}\left(a_{m+2} a_{m+1} \cdots a_{1}\right) / S \text {. }
$$

Then $r_{R}\left(a_{m+1} a_{m} \cdots a_{1}\right)=r_{R}\left(a_{m+2} a_{m+1} \cdots a_{1}\right)$, and so $\left(a_{m+1} a_{m} \cdots a_{1}\right) R \cap$ $r_{R}\left(a_{m+2}\right)=0$. Note that $r_{R}\left(a_{m+2}\right)$ is also an essential right ideal of $R$, then $a_{m+1} a_{m} \cdots a_{1}=0$. So $J(R)$ is a right $T$-nilpotent ideal and the ideal $J(R)+$ $S / S$ of $\bar{R}$ is also a right $T$-nilpotent. By [1, Proposition 29.1], $J(R)+S / S$ is nilpotent. Then there exists a positive integer $t$ such that $(J(R))^{t} \subseteq S$, so $(J(R))^{t+1} \subseteq J(R) S=0$, as desired.

Proposition 2.16. If $R$ is a right APS-injective (resp. PS-injective, APinjective) ring, so is eRe for all $e^{2}=e \in R$ such that $R e R=R$.

Proof. Let $S=e R e$ and let $a \in J(S)=e J e$. Then $a=a e \in J(R)$, so there exists a left ideal $X_{a}$ of $R$ such that $l_{R} r_{R}(a)=R a \oplus X_{a}$. Since $1-e \in r_{R}(a)$, we see that $t(1-e)=0$ for any $t \in X_{a}$, which implies $X_{a}=X_{a} e$. Thus $e R a e \cap e X_{a} e=0$. Clearly, $e R a e \subseteq l_{S} r_{S}(a)$ and $e X_{a} e \subseteq l_{S} r_{S}(a)$ since $R a e=R a$ and $X_{a} e=X_{a}$. Now we prove the other inclusion. Take $x \in l_{S} r_{S}(a)$, and write $1=\sum_{i=1}^{n} a_{i} e b_{i}$ for some $a_{i}, b_{i}$ in $R$. Then for any $y \in r_{R}(a)$, we get aeya $_{i} e=$ aya $_{i} e=0$ for each $i$. This implies that $x e y a_{i} e=0$ for each $i$, which gives $x y=x e y=x e y \sum_{i=1}^{n} a_{i} e b_{i}=0$ since $x \in S$. So $x \in l_{R} r_{R}(a)$, and hence $l_{S} r_{S}(a) \subseteq l_{R} r_{R}(a)$. Take $x=s+t$, where $s \in R a$ and $t \in X_{a}$. Hence, $x=$ exe $=$ $e s e+e t e \in e R a e+e X_{a} e$. This shows that $l_{S} r_{S}(a)=e R a e \oplus e X_{a} e=S a \oplus e X_{a}$, where $e X_{a}$ is a left ideal of $S$. Therefore, $S$ is right $A P S$-injective.

Remark 2.17. The condition that $R e R=R$ in Proposition 2.16 is needed. For example, let $R$ be the algebra of matrices, over a field $F$, of the form

$$
R=\left(\begin{array}{cccccc}
a & x & 0 & 0 & 0 & 0 \\
0 & b & 0 & 0 & 0 & 0 \\
0 & 0 & c & y & 0 & 0 \\
0 & 0 & 0 & a & 0 & 0 \\
0 & 0 & 0 & 0 & b & z \\
0 & 0 & 0 & 0 & 0 & c
\end{array}\right)
$$

By [5, Example 9], $R$ is a $Q F$-ring, and hence it is right $A P S$-injective. Let $e=e_{11}+e_{22}+e_{44}+e_{55}$ be a sum of canonical matrix units. Then $e$ is an idempotent of $R$ such that $R e R \neq R$ and $e R e \cong S=\left(\begin{array}{cc}F & F \\ 0 & F\end{array}\right)$. We claim that $S$ is not right $A P S$-injective. In fact, $J(S)=\left(\begin{array}{ll}0 & F \\ 0 & 0\end{array}\right)$. Then for any $\bar{d}=\left(\begin{array}{ll}0 & d \\ 0 & 0\end{array}\right) \in J(S)$, $l_{S} r_{S}(\bar{d})=\left(\begin{array}{ll}0 & F \\ 0 & F\end{array}\right)$ and $S \bar{d}=\left(\begin{array}{ll}0 & F \\ 0 & 0\end{array}\right)$. So it does not exist a left ideal $X_{\bar{d}}$ of $S$ such that $l_{S} r_{S}(\bar{d})=S \bar{d} \oplus X_{\bar{d}}$.

Corollary 2.18. If the matrix ring $M_{n}(R)$ over a ring $R$ is right $A P S$-injective $(n \geq 1)$, then so is $R$.

Proof. If $S=M_{n}(R)$ is right $A P S$-injective, so is $R \cong e_{11} S e_{11}$ by Proposition 2.16 because $S e_{11} S=S$ (here $e_{i j}$ is the matrix unit). 
We do not know if the converse of Corollary 2.18 holds. However, we have the following result motivated by [11, Theorem 3.8].

Theorem 2.19. Let $R$ be a ring and $n \geq 1$. Then the following are equivalent:

(1) $M_{n}(R)$ is right APS-injective.

(2) $\operatorname{Hom}_{R}\left(R^{n}, R\right)$ is a direct summand of $\operatorname{Hom}_{R}(I, R)$ as left $R$-modules for any $n$-generated $R$-submodule $I$ of $J^{n}$.

Proof. $(1) \Rightarrow(2)$. Let $S=M_{n}(R)$ and let $I=\overline{a_{1}} R+\cdots+\overline{a_{n}} R \in J^{n}$. Write $\left(\overline{a_{1}}, \ldots, \overline{a_{n}}\right)=A$, then $A \in J(S)$. By hypothesis, we have $l_{S} r_{S}(A)=S A \oplus X_{A}$ for some left ideal $X_{A}$ of $S$. Let

$$
\Gamma=\left\{f \in \operatorname{Hom}_{R}(I, R):\left(\begin{array}{ccc}
f\left(\overline{a_{1}}\right) & \cdots & f\left(\overline{a_{n}}\right) \\
0 & \cdots & 0 \\
\vdots & & \vdots \\
0 & \cdots & 0
\end{array}\right) \in X_{A}\right\} .
$$

It is easy to verify that $\Gamma$ is a left $R$-submodule of $\operatorname{Hom}_{R}(I, R)$. We claim that $\operatorname{Hom}_{R}(I, R)=\operatorname{Hom}_{R}\left(R^{n}, R\right) \oplus \Gamma$ as left $R$-modules. In fact, for any $g \in \operatorname{Hom}_{R}(I, R)$, write

$$
B=\left(\begin{array}{ccc}
g\left(\overline{a_{1}}\right) & \cdots & g\left(\overline{a_{n}}\right) \\
0 & \cdots & 0 \\
\vdots & & \vdots \\
0 & \cdots & 0
\end{array}\right)
$$

Then $B \in l_{S} r_{S}(A)$, and hence $B=\left(c_{i j}\right) A+\left(d_{i j}\right)$, where $\left(c_{i j}\right) \in S$ and $\left(d_{i j}\right) \in$ $X_{A}$. Let $h: R^{n} \rightarrow R, \sum_{i=1}^{n} \overline{e_{i}} r_{i} \mapsto \sum_{i=1}^{n} c_{1 i} r_{i}$, where $\overline{e_{i}}$ is the standard basis of $R^{n}$ over $R$, and let $k: I \rightarrow R, \sum_{i=1}^{n} \overline{a_{i}} r_{i} \mapsto \sum_{i=1}^{n} d_{1 i} r_{i}$. Then $g=h+k$. Note that

$$
\left(\begin{array}{ccc}
d_{11} & \cdots & d_{1 n} \\
0 & \cdots & 0 \\
\vdots & & \vdots \\
0 & \cdots & 0
\end{array}\right)=\left(\begin{array}{ccc}
1 & \cdots & 0 \\
0 & \cdots & 0 \\
\vdots & & \vdots \\
0 & \cdots & 0
\end{array}\right)\left(d_{i j}\right) \in X_{A}
$$

So $k \in \Gamma$. Therefore, we have $\operatorname{Hom}_{R}(I, R)=\operatorname{Hom}_{R}\left(R^{n}, R\right)+\Gamma$. Suppose $l \in \operatorname{Hom}_{R}\left(R^{n}, R\right) \cap \Gamma$. Then there exists $\left(c_{1}, \ldots, c_{n}\right) \in R^{n}$ such that $\left(l\left(\overline{a_{1}}\right), \ldots\right.$, $\left.l\left(\overline{a_{n}}\right)\right)=\left(c_{1}, \ldots, c_{n}\right) A$. Thus,

$$
\left(\begin{array}{ccc}
l\left(\overline{a_{1}}\right) & \cdots & l\left(\overline{a_{n}}\right) \\
0 & \cdots & 0 \\
\vdots & & \vdots \\
0 & \cdots & 0
\end{array}\right)=\left(\begin{array}{ccc}
c_{1} & \cdots & c_{n} \\
0 & \cdots & 0 \\
\vdots & & \vdots \\
0 & \cdots & 0
\end{array}\right) A \in S A \cap X_{A}=0
$$

Therefore, $\operatorname{Hom}_{R}(I, R)=\operatorname{Hom}_{R}\left(R^{n}, R\right) \oplus \Gamma$. 
$(2) \Rightarrow(1)$. Suppose $A=\left(a_{i j}\right) \in J(S)$. Let $I=\overline{a_{1}} R+\cdots+\overline{a_{n}} R$, where $\overline{a_{i}}$ is $i$-th column of $A$. Then $I \in J^{n}$. By hypothesis, we have $\operatorname{Hom}_{R}(I, R)=$ $\operatorname{Hom}_{R}\left(R^{n}, R\right) \oplus \Gamma$ for some left $R$-submodule $\Gamma$ of $\operatorname{Hom}_{R}(I, R)$. Let

$$
X_{A}=\left\{\left(\begin{array}{ccc}
f_{1}\left(\overline{a_{1}}\right) & \cdots & f_{1}\left(\overline{a_{n}}\right) \\
f_{2}\left(\overline{a_{1}}\right) & \cdots & f_{2}\left(\overline{a_{n}}\right) \\
\vdots & & \vdots \\
f_{n}\left(\overline{a_{1}}\right) & \cdots & f_{n}\left(\overline{a_{n}}\right)
\end{array}\right): f_{i} \in \Gamma, i=1,2, \ldots, n\right\} .
$$

Then $X_{A}$ is a left ideal of $S$. Now we show that $l_{S} r_{S}(A)=S A \oplus X_{A}$ as left $S$-modules. It is easy to check that $X_{A} \subseteq l_{S} r_{S}(A)$. If $B=\left(b_{i j}\right) \in$ $l_{S} r_{S}(A)$, then $r_{S}(A) \subseteq r_{S}(B)$. So $f: A S \rightarrow B S, A\left(s_{i j}\right) \mapsto B\left(s_{i j}\right),\left(s_{i j}\right) \in S$ is a well-defined $S$-homomorphism, which induces an $R$-homomorphism $f_{i}$ : $\sum_{j=1}^{n} \overline{a_{j}} r_{j} \mapsto \sum_{j=1}^{n} b_{i j} r_{j}$ from $I$ to $R$ for each $1 \leq i \leq n$. Write $f_{i}=$ $g_{i}+h_{i}$, where $g_{i} \in \operatorname{Hom}_{R}\left(R^{n}, R\right)$ and $h_{i} \in \Gamma$. Then, for each $i$, there exists $\left(c_{i 1}, \ldots, c_{i n}\right) \in R^{n}$ such that $\left(g_{i}\left(\overline{a_{1}}\right), \ldots, g_{i}\left(\overline{a_{n}}\right)\right)=\left(c_{i 1}, \ldots, c_{i n}\right) A$. So,

$$
B=\left(b_{i j}\right)=\left(c_{i j}\right) A+\left(\begin{array}{ccc}
h_{1}\left(\overline{a_{1}}\right) & \cdots & h_{1}\left(\overline{a_{n}}\right) \\
h_{2}\left(\overline{a_{1}}\right) & \cdots & h_{2}\left(\overline{a_{n}}\right) \\
\vdots & & \vdots \\
h_{n}\left(\overline{a_{1}}\right) & \cdots & h_{n}\left(\overline{a_{n}}\right)
\end{array}\right) \in S A+X_{A},
$$

showing $l_{S} r_{S}(A)=S A+X_{A}$. Let $C \in S A \cap X_{A}$. Then for some $\left(d_{i j}\right) \in S$ and some $k_{i} \in \Gamma(i=1,2, \ldots, n)$,

$$
C=\left(\begin{array}{ccc}
k_{1}\left(\overline{a_{1}}\right) & \cdots & k_{1}\left(\overline{a_{n}}\right) \\
k_{2}\left(\overline{a_{1}}\right) & \cdots & k_{2}\left(\overline{a_{n}}\right) \\
\vdots & & \vdots \\
k_{n}\left(\overline{a_{1}}\right) & \cdots & k_{n}\left(\overline{a_{n}}\right)
\end{array}\right) \in\left(d_{i j}\right) A
$$

Then, for each $i,\left(k_{i}\left(\overline{a_{1}}\right), \ldots, k_{i}\left(\overline{a_{n}}\right)\right)=\left(d_{i 1}, \ldots, d_{i n}\right) A$, which shows that $k_{i} \in$ $\operatorname{Hom}_{R}\left(R^{n}, R\right) \cap \Gamma=0$. Thus, each $k_{i}=0$, and hence $C=0$. Therefore, $l_{S} r_{S}(A)=S A \oplus X_{A}$.

The following theorem is a generalization of [17, Theorem 2.1].

Theorem 2.20. Let $R$ be a right Noetherian, left APS-injective ring. Then

(1) $l_{R}(J) \leq{ }_{R} R$.

(2) $J$ is nilpotent.

(3) $l_{R}(J) \leq{ }^{e} R_{R}$.

Proof. (1) For any $0 \neq x \in R$, it is enough to show that $l_{R}(J) \cap R x \neq 0$. Since $R$ has $A C C$ on right annihilators, choose $y \in R$ such that $y x \neq 0$ and $r_{R}(y x)$ is maximal in $\left\{r_{R}(a x) \mid a \in R, a x \neq 0\right\}$. Now we prove that $y x J=0$. Otherwise, there exists a $t \in J$ such that $y x t \neq 0$. Note that $y x t \in J$ and $R$ is left $A P S$-injective, then $r_{R} l_{R}(y x t)=y x t R \oplus X$ for some right ideal $X$ of $R$. We proceed with the following two cases. 
Case 1. $r_{R} l_{R}(y x)=r_{R} l_{R}(y x t)$. Then $y x \in r_{R} l_{R}(y x t)=y x t R \oplus X$. Write $y x=y x t r+z$, where $y x \operatorname{tr} \in y x t R-X$ and $z \in X-y x t R$. So $z=y x(1-t r)$, and hence $y x=z(1-t r)^{-1}$ since $1-t r$ is invertible, contradicting with yxtr $\notin X$.

Case 2. $r_{R} l_{R}(y x) \neq r_{R} l_{R}(y x t)$. Then $l_{R}(y x) \neq l_{R}(y x t)$. It follows that there exists $u \in l_{R}(y x t)$ but $u \notin l_{R}(y x)$. Thus $u y x t=0$ and $u y x \neq 0$. This gives that $t \in r_{R}(u y x)$ and $t \notin r_{R}(y x)$. So $r_{R}(y x) \subset r_{R}(u y x)$, contradicting the maximality of $r_{R}(y x)$.

Then $y x J=0$, and so $0 \neq y x \in l_{R}(J) \cap R x$. Therefore, $l_{R}(J) \leq{ }_{R} R$.

(2) There exists $k \geq 1$ such that $l_{R}\left(J^{k}\right)=l_{R}\left(J^{k+1}\right)=\cdots$. If $J$ is not nilpotent, choose $r_{R}(x)$ to be maximal in $\left\{r_{R}(y) \mid y J^{k} \neq 0\right\}$. Then $x J^{2 k} \neq$ 0 because $l_{R}\left(J^{2 k}\right)=l_{R}\left(J^{k}\right)$, so there exists $b \in J^{k}$ with $x b J^{k} \neq 0$. Since $l_{R}(J) \leq l_{R}\left(J^{k}\right)$, we have $l_{R}\left(J^{k}\right) \leq{ }_{R} R$ by (1). Thus, $R x b \cap l_{R}\left(J^{k}\right) \neq 0$, say $0 \neq c x b \in l_{R}\left(J^{k}\right)$. Hence, $r_{R}(x) \subset r_{R}(c x)$ because $x b J^{k} \neq 0$, contradicting the maximality of $r_{R}(x)$.

(3) If $0 \neq d \in R$, we must show that $d R \cap l_{R}(J) \neq 0$. It is clear if $d J=0$. Otherwise, since $J$ is nilpotent by (2), there exists $m \geq 1$ such that $d J^{m} \neq 0$ but $d J^{m+1}=0$. Then $0 \neq d J^{m} \subseteq d R \cap l_{R}(J)$, as desired.

In [17], a module $M$ is said to satisfy the generalized C2-condition (GC2) if, for any $N \subseteq M$ and $N \cong M, N$ is a summand of $M$.

Corollary 2.21. If $R$ is a right Noetherian, left APS-injective ring such that $R_{R}$ satisfies (GC2), then it is right Artinian.

Proof. Note that $R$ is right finitely dimensional. By [17, Lemma 1.1], $R$ is semilocal. By Theorem 2.18, $J(R)$ is nilpotent. Thus, $R$ is semiprimary, and hence it is right Artinian by the Hopkins-Levitzki theorem.

The condition that $R_{R}$ satisfies (GC2) can not be omitted. For example, the ring $R=\mathbb{Z}$ is a Noetherian and $A P S$-injective ring but not Artinian. From [10, Proposition 1.46], a left Kasch ring is right $C 2$. Thus, we have the following corollary.

Corollary 2.22. If $R$ is a right Noetherian, left Kasch and left APS-injective ring, then it is right Artinian.

\section{Trivial extensions}

Let $R$ be a ring and $M$ a bimodule over $R$. The trivial extension of $R$ and $M$ is

$$
R \propto M=\{(a, x) \mid a \in R, x \in M\}
$$

with addition defined componentwise and multiplication defined by

$$
(a, x)(b, y)=(a b, a y+x b) .
$$

For convenience, we write $I \propto X=\{(a, x) \mid a \in I, x \in X\}$, where $I$ is a subset of $R$ and $X$ is a subset of $M$. It is easy to check that $J(R \propto M)=J(R) \propto M$. 
Proposition 3.1. Let $R$ be a ring and $X_{a}$ a left ideal of $R$ for any $a \in R$, $S=R \propto R$. Then the following are equivalent:

(1) $l_{R} r_{R}(a)=R a \oplus X_{a}$.

(2) $l_{S} r_{S}(0, a)=S(0, a) \oplus X_{(0, a)}$, where $X_{(0, a)}=0 \propto X_{a}$ is a left ideal of $S$.

(3) $l_{S} r_{S}(a, 0)=S(a, 0) \oplus X_{(a, 0)}$, where $X_{(a, 0)}=X_{a} \propto 0$ is a left ideal of $S$.

(4) $l_{S} r_{S}(a, a)=S(a, a) \oplus X_{(a, a)}$, where $X_{(a, a)}=X_{a} \propto X_{a}$ is a left ideal of $S$.

Proof. $(1) \Rightarrow(2)$. For any $(b, c) \in l_{S} r_{S}(0, a), r_{S}(0, a) \subseteq r_{S}(b, c)$. Since $(0,1) \in$ $r_{S}(0, a), 0=(b, c)(0,1)=(0, b)$, showing $b=0$. If $x \in r_{R}(a)$, then $(x, 0) \in$ $r_{S}(0, a) \subseteq r_{S}(b, c)$, showing that $0=(0, c)(x, 0)=(0, c x)$. So $x \in r_{R}(c)$, and hence $r_{R}(a) \subseteq r_{R}(c)$. Thus, $c \in l_{R} r_{R}(c) \subseteq l_{R} r_{R}(a)=R a \oplus X_{a}$. Write $c=r a+y$, where $r a \in R a-X_{a}$ and $y \in X_{a}-R a$. Then $(b, c)=(0, r a+y)=$ $(r, 0)(0, a)+(0, y) \in S(0, a)+X_{(0, a)}$, where $X_{(0, a)}=0 \propto X_{a}$ is a left ideal of $S$. It is easy to prove that $S(0, a) \cap X_{(0, a)}=0$, so $l_{S} r_{S}(0, a) \subseteq S(0, a) \oplus X_{(0, a)}$. Conversely, for any $(m, n) \in S(0, a) \oplus X_{(0, a)}$, where $X_{(0, a)}=0 \propto X_{a}$ is a left ideal of $S$. Then $(m, n)=\left(r_{1}, r_{2}\right)(0, a)+(0, y)=\left(0, r_{1} a+y\right)$, where $\left(r_{1}, r_{2}\right)(0, a) \in S(0, a)-X_{(0, a)}$ and $(0, y) \in X_{(0, a)}-S(0, a)$. Note that $r_{1} a \in$ $R a-X_{a}$ and $y \in X_{a}-R a$, so $m=0, n=r_{1} a+y \in R a \oplus X_{a}=l_{R} r_{R}(a)$. Then $r_{R}(a) \subseteq r_{R}(n)$. For any $(k, l) \in r_{S}(0, a), 0=(0, a)(k, l)=(0, a k)$, showing $k \in r_{R}(a)$, and hence $n k=0$. Then $(m, n)(k, l)=(0, n)(k, l)=(0, n k)=0$, proving $(m, n) \in l_{S} r_{S}(0, a)$.

$(2) \Rightarrow(1)$. For any $b \in l_{R} r_{R}(a), r_{R}(a) \subseteq r_{R}(b)$. If $(x, y) \in r_{S}(0, a)$, then $a x=0$. So $x \in r_{R}(a) \subseteq r_{R}(b)$, showing $(0, b)(x, y)=0$. Thus, $(x, y) \in$ $r_{S}(0, b)$. So $r_{S}(0, a) \subseteq r_{S}(0, b)$. This gives that $(0, b) \in l_{S} r_{S}(0, b) \subseteq l_{S} r_{S}(0, b)=$ $S(0, a) \oplus X_{(0, a)}$. Write $(0, b)=\left(r_{1}, r_{2}\right)(0, a)+(0, y)=\left(0, r_{1} a+y\right)$, where $\left(r_{1}, r_{2}\right)(0, a) \in S(0, a)-X_{(0, a)}$ and $(0, y) \in X_{(0, a)}-S(0, a)$. Note that $r_{1} a \in$ $R a-X_{a}$ and $y \in X_{a}-R a$, so $b=r_{1} a+y \in R a \oplus X_{a}$, proving $l_{R} r_{R}(a) \subseteq R a \oplus X_{a}$. Now we show the other inclusion. For any $c \in R a \oplus X_{a}$, write $c=r a+z$, where $r a \in R a-X_{a}$ and $z \in X_{a}-R a$. Then $(0, c)=(0, r a)+(0, z)=$ $(r, 0)(0, a)+(0, z) \in S(0, a) \oplus X_{(0, a)}=l_{S} r_{S}(0, a)$. So $r_{S}(0, a) \subseteq r_{S}(0, c)$. If $x \in r_{R}(a)$, then $(x, 0) \in r_{S}(0, a)$, showing $0=(0, c)(x, 0)=(0, c x)$, and hence $x \in r_{R}(c)$. Thus, $r_{R}(a) \subseteq r_{R}(c)$. This implies that $c \in l_{R} r_{R}(c) \subseteq l_{R} r_{R}(a)$.

The proofs of $(1) \Leftrightarrow(3)$ and $(1) \Leftrightarrow(4)$ are similar to that of $(1) \Leftrightarrow(2)$.

Corollary 3.2. Let $R$ be a ring and $a \in R, S=R \propto R$. Then the following are equivalent:

(1) $l_{R} r_{R}(a)=R a$.

(2) $l_{S} r_{S}(0, a)=S(0, a)$.

(3) $l_{S} r_{S}(a, 0)=S(a, 0)$.

(4) $l_{S} r_{S}(a, a)=S(a, a)$. 
Corollary 3.3. Let $R$ be a ring. If $R \propto R$ is right $A P S$-injective, then $R$ is right AP-injective.

Proof. Let $S=R \propto R$. For any $0 \neq a \in R,(0, a) \in J(S)$. So there exists a left ideal $X_{(0, a)}$ of $S$ such that $l_{S} r_{S}(0, a)=S(0, a) \oplus X_{(0, a)}$. By the proof of $(1) \Rightarrow(2)$ in Proposition 3.1, if $(b, c) \in l_{S} r_{S}(0, a)$ and $(m, n) \in S(0, a)$, then $b=0$ and $m=0$. So $X_{(0, a)}=0 \propto X_{a}$, where $X_{a}$ is a left ideal of $R$. By Proposition 3.1 again, we have $l_{R} r_{R}(a)=R a \oplus X_{a}$, proving that $R$ is right $A P$-injective.

Remark 3.4. We claim that $R$ being right $A P S$-injective can not imply $R \propto R$ being right $A P S$-injective. For example, let $R=\mathbb{Z}$ be the ring of integers. Suppose that $S=\mathbb{Z} \propto \mathbb{Z}$ is $A P S$-injective, then $\mathbb{Z}$ is $A P$-injective by Corollary 3.3 , a contradiction.

For $f, g \in \operatorname{Hom}_{R}(R, R)$, define $\alpha=(f, g)$ such that $\alpha(a, b)=(f, g)(a, b)=$ $(f(a), f(b)+g(a))$, where $(a, b) \in S=R \propto R$. It is easy to check that $\alpha \in$ $\operatorname{Hom}_{S}(S, S)$. Conversely, for any $\alpha \in \operatorname{Hom}_{S}(S, S)$, let $\alpha(1,0)=(p, q)$. Define $f(1)=p, g(1)=q$, then $f, g \in \operatorname{Hom}_{R}(R, R)$ and $\alpha=(f, g)$. In the following theorem, we shall discuss when $R \propto R$ is right $A P S$-injective.

Theorem 3.5. Let $R$ be a ring. If, for any $a \in J(R), b \in R, \operatorname{Hom}_{R}(a R+$ $\left.b r_{R}(a), R\right)=\operatorname{Hom}_{R}(R, R) \oplus X$ as left $R$-modules for some submodules $X$ of $\operatorname{Hom}_{R}\left(a R+b r_{R}(a), R\right)$, then $R \propto R$ is right $A P S$-injective.

Proof. Let $S=R \propto R$ and any $A \in J(S)$. By Lemma 2.2, it is enough to show that $\operatorname{Hom}_{S}(A S, S)=\operatorname{Hom}_{S}(S, S) \oplus Y$ for some left $S$-submodules $Y$ of $\operatorname{Hom}_{S}(A S, S)$. Write $A=(a, b)$, then $a \in J(R), b \in R$. For any $f \in$ $\operatorname{Hom}_{S}(A S, S)$, say $f(A)=(p, q), p, q \in R$. Define $g: a R+b r_{R}(a) \rightarrow R, a r_{1}+$ $b r_{2} \mapsto p r_{1}+q r_{2}$. If $a r_{1}+b r_{2}=0$, then $(a, b)\left(r_{2}, r_{1}\right)=\left(a r_{2}, a r_{1}+b r_{2}\right)=0$ since $a r_{2}=0$, and hence $0=f\left((a, b)\left(r_{2}, r_{1}\right)\right)=(p, q)\left(r_{2}, r_{1}\right)=\left(p r_{2}, p r_{1}+q r_{2}\right)$, which implies $p r_{1}+q r_{2}=0$. So $g \in \operatorname{Hom}_{R}\left(a R+b r_{R}(a), R\right)$. By hypothesis, $g=h \oplus k$, where $h \in \operatorname{Hom}_{R}(R, R)$ and $k \in X$. In particular, $p=g(a)=$ $h(a)+k(a)=h(1) a+k(a)$.

If $a=0$, then $r_{R}(a)=R$. So $R$ is right $A P$-injective. Define $l: a R \rightarrow$ $R, a r \mapsto q r-h(1) b r-k(b r), r \in R$. If $a r=0$, then $r \in r_{R}(a)$, so $h(1) b r=$ $h(b r)=g(b r)-k(b r)=q r-k(b r)$, and hence $q r-h(1) b r-k(b r)=0$. Thus, $l \in \operatorname{Hom}_{R}(a R, R)$. Then $l=h^{\prime} \oplus k^{\prime}$, where $h^{\prime} \in \operatorname{Hom}_{R}(R, R)$ and $k^{\prime} \in K \subseteq$ $\operatorname{Hom}_{R}(a R, R)$. We have $q-h(1) b-k(b)=l(a)=h^{\prime}(a)+k^{\prime}(a)$, so $q=h(1) b+$ $k(b)+h^{\prime}(1) a+k^{\prime}(a)$. Then $f(A)=(p, q)=\left(h(1) a+k(a), h(1) b+k(b)+h^{\prime}(1) a+\right.$ $\left.k^{\prime}(a)\right)=\left(h(1), h^{\prime}(1)\right)(a, b)+\left(k(a), k(b)+k^{\prime}(a)\right)=\left(h, h^{\prime}\right)(a, b)+\left(k, k^{\prime}\right)(a, b)=$ $\left(h, h^{\prime}\right) A+\left(k, k^{\prime}\right) A$. Note that $\left(h, h^{\prime}\right) \in \operatorname{Hom}_{S}(S, S)$ and $\left(k, k^{\prime}\right) \in Y=\{(i, j) \mid i \in$ $X, j \in K\} \subseteq \operatorname{Hom}_{S}(A S, S)$, then $\operatorname{Hom}_{S}(A S, S) \subseteq \operatorname{Hom}_{S}(S, S)+Y$. Now we show that $\operatorname{Hom}_{S}(S, S) \cap Y=0$. For any $y \in \operatorname{Hom}_{S}(S, S) \cap Y$, write $y=(m, n)$, then $m \in \operatorname{Hom}_{R}(R, R) \cap X=0$ and $n \in \operatorname{Hom}_{R}(R, R) \cap K=0$, proving $y=0$. Therefore, $\operatorname{Hom}_{S}(A S, S) \subseteq \operatorname{Hom}_{S}(S, S) \oplus Y$. But the other inclusion is clear, as desired. 

laries.

Following the preceding theorem, we immediately deduce the following corol-

Corollary 3.6. Let $R$ be a ring. If, for any $a, b \in R, \operatorname{Hom}_{R}\left(a R+b r_{R}(a), R\right)=$ $\operatorname{Hom}_{R}(R, R) \oplus X$ as left $R$-modules for some submodules $X$ of $\operatorname{Hom}_{R}(a R+$ $\left.b r_{R}(a), R\right)$, then $R \propto R$ is right $A P$-injective.

Corollary 3.7. Let $R$ be a ring. If, for any $a \in J(R), b \in R$, any $R$ homomorphism $a R+b \cdot r(a) \rightarrow R$ can be extended to $R$, then $R \propto R$ is right $P S$-injective.

Corollary 3.8. If $R$ is semiprimitive and right $A P$-injective, then $R \propto R$ is right APS-injective.

Remark 3.9. As Remark 3.4, the condition that $R$ is right $A P$-injective in Corollary 3.8 can not be omitted.

\section{References}

[1] F. W. Anderson and K. R. Fuller, Rings and Categories of Modules, Springer-Verlag, New York, 1974.

[2] J. L. Chen and N. Q. Ding, On regularity of rings, Algebra Colloq. 8 (2001), no. 3, $267-274$.

[3] J. L. Chen and Y. Q. Zhou, GP-injective rings need not be P-injective, Comm. Algebra 33 (2005), no. 7, 2395-2402.

[4] _ Extensions of injectivity and coherent rings, Comm. Algebra 34 (2006), no. 1, $275-288$.

[5] K. Koike, Dual rings and cogenerator rings, Math. J. Okayama Univ. 37 (1995), 99-103.

[6] T. Y. Lam, A First Course in Noncommutative rings, Graduate Texts in Mathematic 131, Springer-Verlag, 2001.

[7] L. X. Mao, N. Q. Ding, and W. T. Tong, New characterizations and generalizations of PP-rings, Vietnam J. Math. 33 (2005), no. 1, 97-110.

[8] W. K. Nicholson and F. M. Yousif, Principally injective rings, J. Algebra 174 (1995), no. $1,77-93$.

[9] - Mininjective rings, J. Algebra 187 (1997), no. 2, 548-578.

[10] _ Quasi-Frobenius Rings, Cambridge University Press, Cambridge, 2003.

[11] S. S. Page and Y. Q. Zhou, Generalizations of principally injective rings, J. Algebra 206 (1998), no. 2, 706-721.

[12] L. Shen and J. Chen, New characterizations of quasi-Frobenius rings, Comm. Algebra 34 (2006), no. 6, 2157-2165.

[13] S. Wongwai, Almost mininjective rings, Thai J. Math. 4 (2006), no. 1, 245-249.

[14] Y. M. Xiang, Principally small injective rings, Kyungpook Math. J. 51 (2011), no. 2, $177-185$.

[15] G. S. Xiao, Stable range and regularity of injective rings, (chinese) $\mathrm{Ph}$. D thesis of Nanjing University, 2004.

[16] M. F. Yousif and Y. Q. Zhou, FP-injective, simple-injective, and quasi-Frobenius rings, Comm. Algebra 32 (2004), no. 6, 2273-2285.

[17] Y. Q. Zhou, Rings in which certain right ideals are direct summands of annihilators, J. Aust. Math. Soc. 73 (2002), no. 3, 335-346. 


\section{Yueming Xiang}

Department of Mathematics and Applied Mathematics

Huaihua University

Huainua, 418000, P. R. China

E-mail address: xymls999@126.com 